\title{
Putting $C$. elegans neurons in their place
}

Toyoshima, Y. et al. BMC Biol 18, 30 (2020).

Caenorhabditis elegans is unique among model organisms: the lineages of all 302 of the adult hermaphrodite's neurons have been identified, and their morphologies and connections have been described in atlases for researchers working with the worm to reference. But, individuals may vary.

"In order to compare the properties of neuron $A$ in animal 1 and neuron $B$ in animal 2, we need to know whether neuron A and neuron B have the same identify," commented Yu Toyoshima of the University of Tokyo via email. For example, are they both actually salt-sensing neurons? A given neuron isn't always to be found in the exact same location from adult to adult. Annotation is required, but this process can be time consuming; attempts to automate annotation have been hampered by that variability and limited to small sets of neurons.

Writing in the journal BMC Biology, Toyoshima and his colleagues at the University of Tokyo and the Institute of Statistical Mathematics in Japan describe a resource to improve the neuron identification process in C. elegans.

Focusing on the densely packed neurons in the head region, the team spent several years systematically assembling a volumetric fluorescent image dataset from 311 worms fluorescently tagged with 35 different cell-specific promotors. From expression patterns and neuron position identified in all those animals, the team then developed an algorithm to calculate the optimal combination of promotors for neuron identification, based on the number of cells labeled by particular promotors and the stability, sparseness, and overlap of the resulting expression patterns.

The team then produced 'optimized' worm strains, along with a computerassisted, semi-automated means of assigning the most statically likely identity of each neuron in the head region. With only $60 \%$ accuracy there is still much room to improve. "Increasing the number of colors (fluorescent channels) and increasing the number of promoters will give us more precise neuronal identification," commented Toyoshima. Other information including shape and fluorescent intensity could also help he says-that will just require more complicated image analysis techniques, including deep learning.

Nevertheless, the approach provides a more informed starting point that can make the manual annotation still required more efficient, which Toyoshima and his team are using in their research. "By integrating this resource with the experimental setups for whole-brain activity imaging, we achieved the whole-brain activity data with singlecell neuron identity. Analyzing the data will reveal the information processing mechanisms," Toyoshima commented.

\section{Ellen P. Neff}

Published online: 13 April 2020

https://doi.org/10.1038/s41684-020-0538-9

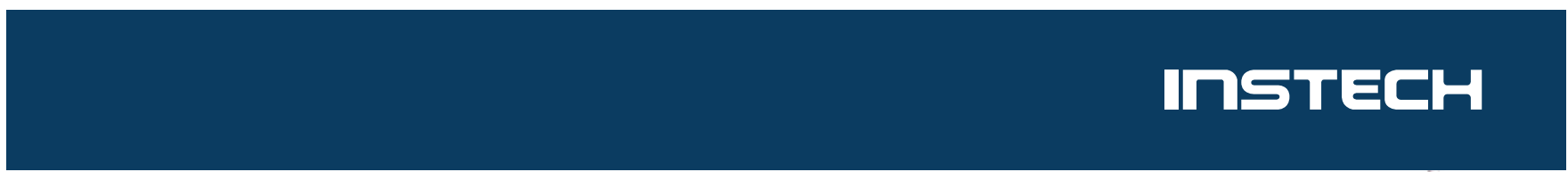

\section{Oral gavage, refined.}

Our plastic feeding needles have flexible tubes with soft tips to reduce trauma, and they're disposable to eliminate the hassle of cleaning.

Available in a range of sizes for mice, rats and other rodents. www.instechlabs.com
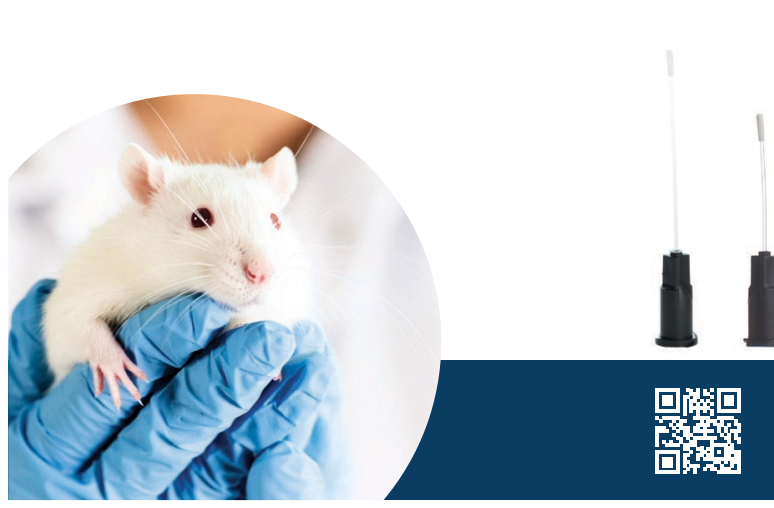